\title{
Efficacy and safety of the Amplatzer Duct Occluder II for ventricular septal defect closure:
}

\section{a meta-analysis}

\author{
Huan Cen'1 , Bo Peng ${ }^{2}$, Jinhua Li' ${ }^{1}$ Sinan Chen'1, Pengtao Sun ${ }^{1}$ \\ 1 Department of Ultrasonography, the Second Affiliated Hospital of Guangzhou University of Chinese \\ Medicine (Guangdong Hospital of Traditional Chinese Medicine), Guangzhou, China \\ 2 Department of Cardiac Surgery, the Second Affiliated Hospital of Guangzhou University of Chinese Medicine \\ (Guangdong Hospital of Traditional Chinese Medicine), Guangzhou, China
}

\section{KEY WORDS}

Amplatzer Duct

Occluder II, congenital

heart disease,

pediatric intervention,

transcatheter closure,

ventricular septal

defect

\section{EDITORIAL}

by Schubert, see p. 378

\section{ABSTRACT}

BACKGROUND Transcatheter closure has become an effective and safe method to repair ventricular septal defects (VSDs). However, the devices used for VSD closure in the past frequently led to serious complications, such as complete atrioventricular block. The second-generation device, the Amplatzer Duct Occluder (ADO II), was originally designed to block small- and medium-sized patent ductus arteriosus. Interestingly, there are some reports of the use of the ADO II to close VSD because of less complications. AIMS A meta-analysis of the literature was performed to systematically investigate the efficacy and safety of the ADO II for VSD closure.

METHODS The Embase, PubMed, Cochrane, and Web of Science databases were searched for original studies on VSD closure with the ADO II up to March 15, 2020. The random-effects model and summary rate were employed to estimate the success and complications of VSD closure with the ADO II.

RESULTS A total of 13 articles comprising 478 patients with VSD were included. The age of the patients ranged from 0.5 to 55.7 years. The overall estimated device-implantation success rate was $99 \%$ (95\% CI, $98 \%-100 \%$ ). Residual shunts (pooled rate, $4 \% ; 95 \% \mathrm{CI}, 1 \%-7 \%$ ) and postoperative aortic valve regurgitation (pooled rate, 0\%; 95\% CI, 0-1\%) were common complications. Only 3 patients developed device embolism. CONCLUSIONS The ADO II may be a safer and more effective transcatheter closure device for patients with VSD due to its higher success rate and lower complication rate, as compared with other devices.

INTRODUCTION Ventricular septal defect (VSD) is a common congenital heart disease that can lead to left ventricular volume overload, resulting in a variety of complications, such as ventricular insufficiency, arrhythmia, aortic regurgitation, pulmonary hypertension, and endocarditis. ${ }^{1,2}$

Although transcatheter closure has become an effective and safe method to repair VSDs, ${ }^{3,4}$ VSD closure with devices used in the past was associated with serious complications, such as complete atrioventricular block (cAVB). Fortunately, the second-generation Amplatzer Duct Occluder (ADO II) appears to be safer for patients with VSD. The ADO II, improved by AGA
Medical, is a modified version of the ADO I. The ADO II was originally designed to block smalland medium-sized patent ductus arteriosus. ${ }^{5}$ Recently, the off-label use of the ADO II for closing VSD has been described. ${ }^{6}$

Due to the finer and more flexible mesh structure of the ADO II, the device can pass through a small $4 \mathrm{~F}$ or $5 \mathrm{~F}$ catheter system. ' In addition, its soft design is expected to reduce the risk of $\mathrm{CAVB} .{ }^{8}$ Indeed, some studies have reported that the ADO II can be used to close VSD in patients of different ages and in various sites, including perimembranous VSD (pmVSD) and muscular VSD (mVSD), with a technically high surgical success rate and a relatively low incidence of complications. ${ }^{9,10}$ 


\section{WHAT'S NEW?}

The Amplatzer Duct Occluder II (ADO II) was originally designed to block smalland medium-sized patent ductus arteriosus, but the off-label use of the ADO II to close ventricular septal defect (VSD) has been described. To the best of our knowledge, this is the first systematic review and meta-analysis to collate the outcomes of VSD closure with the ADO II.

However, studies with small sample sizes may not provide sufficient data to influence clinical practice; for example, Kanaan et a $\mathbf{l}^{8}$ showed a success rate of $93 \%$, but Vijayalakshmi et a $1^{11}$ demonstrated a rate of $100 \%$. Thus, a study with a larger sample size is needed to objectively evaluate the potential value of VSD closure with the ADO II.

Accordingly, the purpose of this study was to calculate the success and complication rates of VSD closure with the ADO II based on a comprehensive analysis of the literature. Our results provide evidence and an important reference for clinical decision-making regarding the use of the ADO II as a VSD occluder. The findings will also guide further research and the ADO II development.

METHODS Search strategy and selection criteria This systematic review and meta-analysis was performed in accordance with the Preferred Reporting Items for Systematic Reviews and Meta-Analyses (PRISMA) Statement and was registered at International Prospective Register of Systematic Reviews (no. CRD42020175385).12 All analyses were based on previously published studies; thus, neither ethical approval nor patient consent was required.

Using the Embase, PubMed, Cochrane, and Web of Science databases, relevant studies published from inception to March 15, 2020 were systematically searched without language restrictions. The following combined text and $\mathrm{MeSH}$

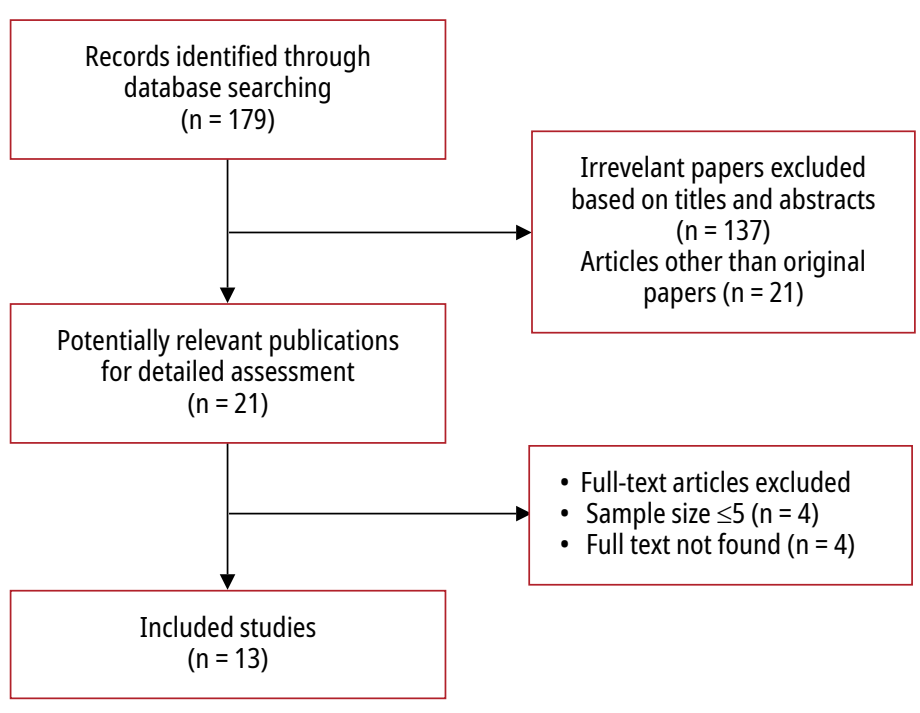

FIGURE 1 Study selection flow terms were used: heart ventricle septum defect and Amplatzer Duct Occluder II. The complete search used for PubMed was as follows: ("Heart Septal Defects, Ventricular" [MeSH] OR Ventric* Sept* Defec*[Title/Abstract] OR Intraventric* Sept* Defec*[Title/Abstract]) AND ("Amplatzer Duct Occlude* II"[Title/Abstract] OR “Amplatzer Duct Occlude* 2" [Title/Abstract] OR "ADO II" [Title/Abstract] OR “ADO 2"[Title/Abstract]). All potentially eligible studies were considered for review, irrespective of the primary outcome or language. A manual search of the references of selected articles published in English was performed. $\mathrm{BP}$ and SC abstracted the data from the selected studies and discrepancies were solved by PS.

Study selection and data extraction The inclusion criteria were as follows: 1) observational or cohort studies; 2) patients with VSD who underwent closure surgery with the ADO II; 3) at least 6 months of follow-up; 4) reporting of the number of successful surgeries and complications, such as residual shunts (RSs), cAVB, device embolism; 5) full-text articles reporting the results of eligible studies; 6) for multiple studies using overlapping samples, only recent ones or those with sufficient data provided were included.

The exclusion criteria were as follows: 1) reviews, editorials, letters, case reports, meeting abstracts, cell and animal studies; 2) insufficient data; 3) sample size of less than 5 patients; and 4) VSD after myocardial infarction in adult patients.

The following data were extracted from each selected study: total number of participants, age, sex, follow-up duration, fluoroscopy time, preoperative pulmonary to systemic blood flow ratio, successful surgeries, and complications. The complications were as follows: RS, cAVB, new or aggravated valve regurgitation after surgery, device embolism, reduced cardiac systolic function, and device thrombosis. The main outcomes are defined below. A successful surgery was defined as a successful implantation without device embolization, implantation of a permanent pacemaker, or secondary surgery due to severe complications. Residual shunt was defined as a shunt around the occluder demonstrated on echocardiography after the surgery. Complete atrioventricular block was diagnosed in case of inability to transmit atrial excitement to the ventricles. Postoperative new or aggravated valve regurgitation was defined as newly discovered or aggravated valve regurgitation after the surgery. Device embolism was understood as embolization caused by device dislodgement to another location in the heart or a blood vessel. A reduced systolic function was defined as left ventricular ejection fraction of less than $50 \%$. Device thrombosis was understood as a thrombus on the occluder or in the catheter insertion route. ${ }^{13}$ 


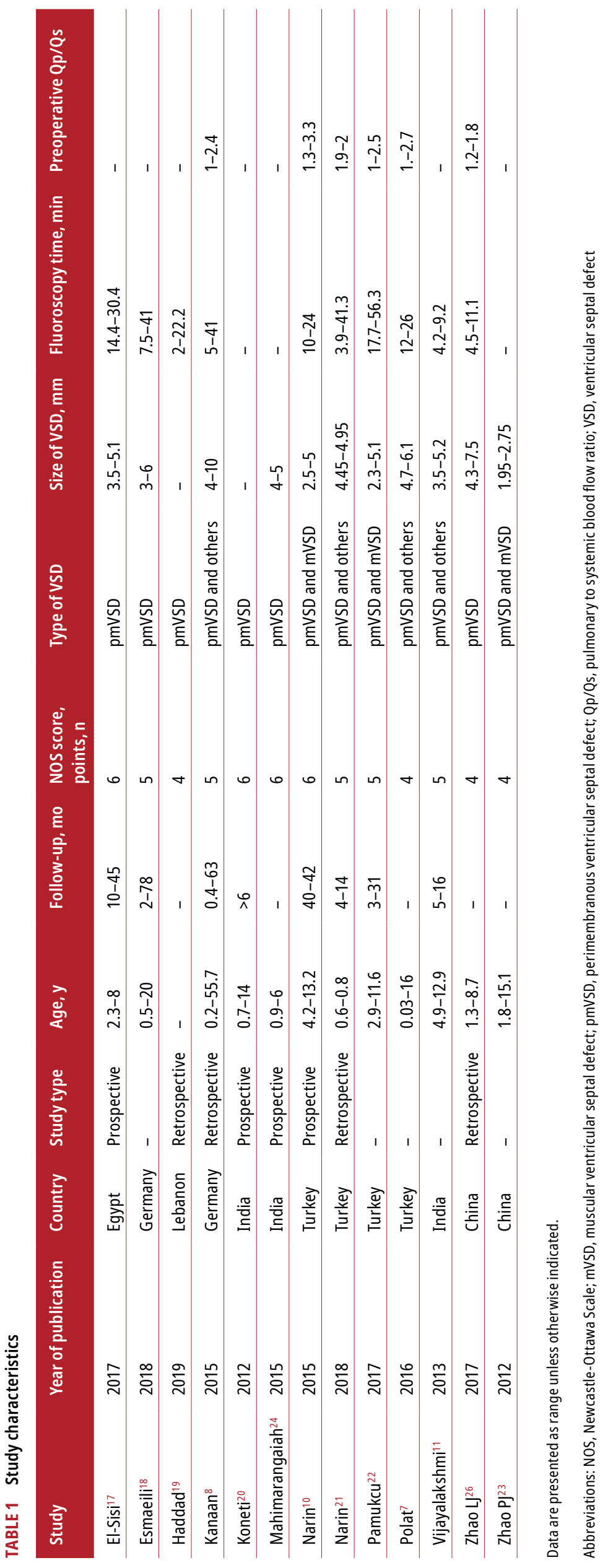

Quality assessment The quality of the included studies was assessed according to the Newcastle-Ottawa Scale (NOS), ${ }^{14}$ in which points are assigned based on cohort selection (0-6), comparability of the groups (0-2), and ascertainment of outcome ( $0-5)$. The NOS scores of 6 or greater indicate high quality.

Statistical analysis For analyses of the proportion of successful cases and cases with complications, we calculated pooled estimates of the incident rate with a random-effects model after double arcsine conversion. Heterogeneity between studies was determined using inconsistency statistics $\left(I^{2}\right)$, whereby an $I^{2}$ value greater than $50 \%$ was considered to indicate substantial heterogeneity. When significant heterogeneity was detected, a subgroup analysis was performed. Sensitivity analyses were also performed by comparing a random-effects model with a fixed-effects model and by excluding each study separately and demonstrating stability of the estimates.

We assessed the possibility of publication bias using Begg and Egger tests, ${ }^{15}$ and we generated an Egger graph. Significant publication bias was defined as $P$ value of less than 0.05 . A trim-and-fill method was applied to provide potential missing studies if publication bias was evident. ${ }^{16}$ STATA version 15.0 (StataCorp LLC, College Station, Texas, United States) was used for all statistical analyses.

RESULTS Study characteristics A total of 150 studies were identified, of which 13 (with data for 478 participants) were included in this analysis (FIGURE 1) 7,8,10,11,17-24: 4 prospective studies, 4 retrospective studies, and 5 studies of uncertain types. The year of publication ranged from 2012 to 2019. The mean follow-up ranged from 6 to 40 months and the mean age of the patients ranged from 0.7 to 8.9 years. General anesthesia was used in the included studies. According to the NOS scores, 4 studies were of high quality (score 26) (tABles 1 and 2).

Outcomes Success rates The success rate of implantation was high. Eight studies achieved a $100 \%$ success rate, and the other 5 studies $^{8,20,23,25,26}$ reported success rates greater than $90 \%$. The inconsistency statistic $\left(I^{2}\right)$ indicated little or no heterogeneity $\left(I^{2}=0 \%\right)$. Thus, the random-effects model was applied, and the pooled estimate of the overall success rate of implantation for the 13 studies (data for 478 participants) was 99\% (95\% CI, 98\%-100\%) (FIGURE2A).

Complication rates Residual shunts The most common complication was RS. A total of 54 patients had RSs immediately after the procedure. However, RS was noted as permanent only in 
TABLE 2 Basic data of the included studies

\begin{tabular}{|c|c|c|c|c|c|c|c|c|c|c|c|}
\hline Author & $\begin{array}{l}\text { Year } \\
\text { of publication }\end{array}$ & Patients & Failure & RS & CAVB & $\begin{array}{l}\text { Device } \\
\text { embolism }\end{array}$ & TR & AR & MR & $\begin{array}{l}\text { Low ejection } \\
\text { fraction }\end{array}$ & $\begin{array}{l}\text { Device } \\
\text { thrombus }\end{array}$ \\
\hline El-Sisi ${ }^{17}$ & 2017 & 17 & 0 & 0 & 0 & 0 & 0 & 0 & 0 & 0 & 0 \\
\hline Esmaeili18 & 2018 & 15 & 0 & 2 & 0 & 0 & 3 & 0 & 0 & 0 & 0 \\
\hline Haddad $^{19}$ & 2019 & 27 & 2 & 2 & 0 & 1 & 0 & 0 & 0 & 0 & 0 \\
\hline Kanaan ${ }^{8}$ & 2015 & 31 & 1 & 3 & 0 & 1 & 0 & 2 & 0 & 0 & 0 \\
\hline Koneti ${ }^{20}$ & 2012 & 57 & 1 & 2 & 0 & 1 & 0 & 0 & 0 & 0 & 0 \\
\hline Mahimarangaiah ${ }^{24}$ & 2015 & 45 & 0 & 3 & 0 & 0 & 0 & 0 & 0 & 0 & 0 \\
\hline Narin $^{10}$ & 2015 & 21 & 0 & 2 & 1 & 0 & 0 & 0 & 0 & 0 & 0 \\
\hline Narin $^{21}$ & 2018 & 12 & 0 & 3 & 1 & 0 & 0 & 0 & 0 & 0 & 0 \\
\hline Pamukcu22 & 2017 & 49 & 0 & 2 & 0 & 0 & 5 & 0 & 0 & 0 & 0 \\
\hline Polat ${ }^{7}$ & 2016 & 26 & 0 & 0 & 0 & 0 & 0 & 0 & 0 & 0 & 0 \\
\hline Vijayalakshmi ${ }^{11}$ & 2013 & 79 & 1 & 0 & 1 & 0 & 0 & 0 & 0 & 0 & 0 \\
\hline Zhao LJ'26 & 2017 & 50 & 2 & 1 & 3 & 0 & 1 & 2 & 0 & 0 & 0 \\
\hline Zhao PJ ${ }^{23}$ & 2012 & 46 & 0 & 3 & 1 & 0 & 0 & 0 & 0 & 0 & 0 \\
\hline
\end{tabular}

Data are presented as number of patients.

Abbreviations: AR, aortic regurgitation; CAVB, complete atrioventricular block; MR, mitral regurgitation; RS, residual shunt; TR, tricuspid regurgitation

21 patients, with a pooled rate of $4 \%(95 \%$ CI, $\left.1 \%-7 \% ; I^{2}=22.75 \%\right)$. Only one patient had significant RS requiring device explantation (FIGURE2B). ${ }^{8}$

Complete atrioventricular block Cardiac dysrhythmias were other common complications, and cAVB was the most important among them. Nonetheless, the rate of $\mathrm{CAVB}$ in the 13 studies was very low, with a pooled estimate of 0\% (95\% CI, $\left.0 \%-1 \% ; I^{2}=0 \%\right)$. Only one patient with cAVB required a permanent pacemaker. ${ }^{21}$

Postoperative valve regurgitation Only 13 patients experienced moderate or severe postoperative valve regurgitation due to restricted movement (4 aortic regurgitations [ARs] and 9 tricuspid

A

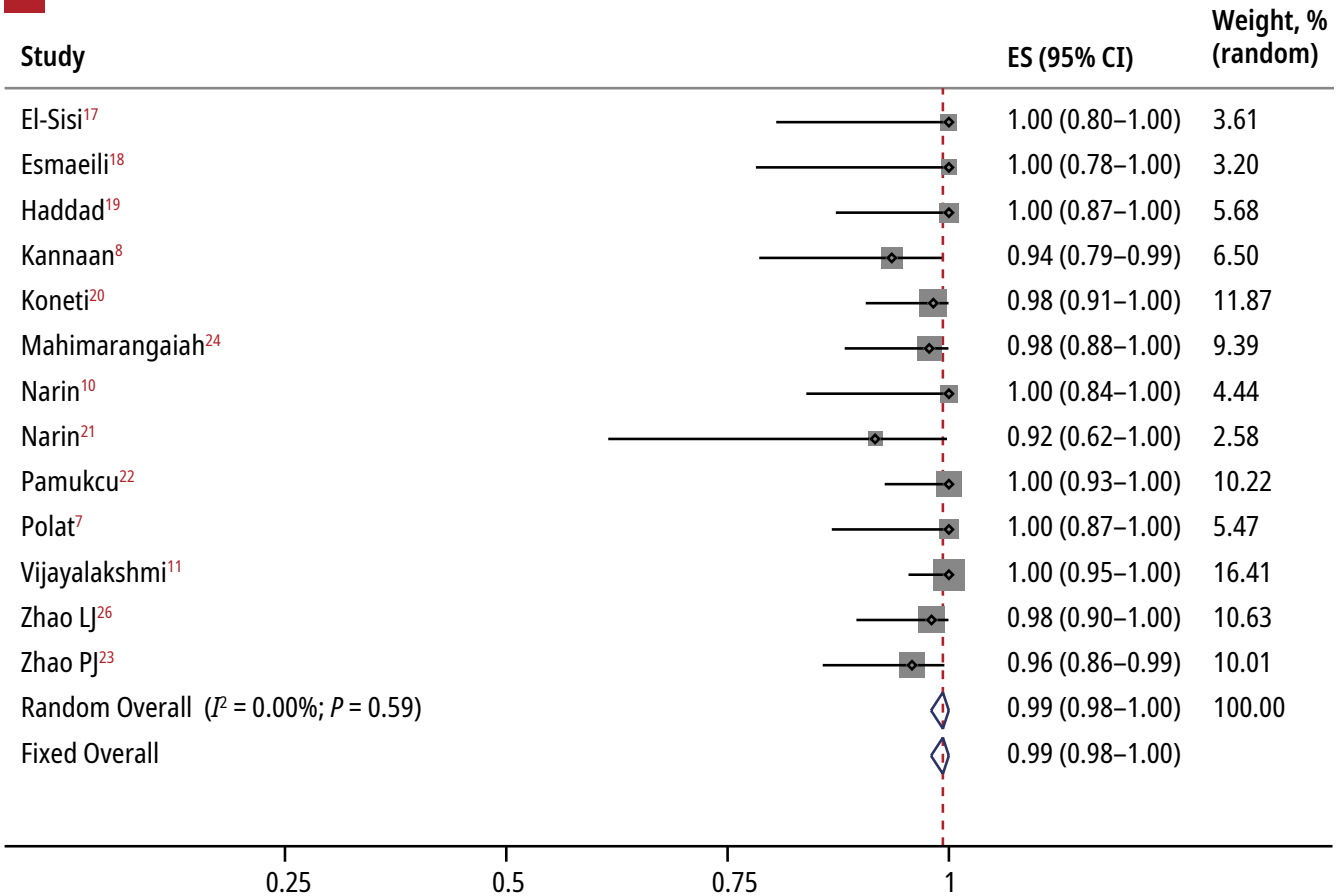

FIGURE 2 A - forest plot of the success rate Abbreviations: ES, effect size 


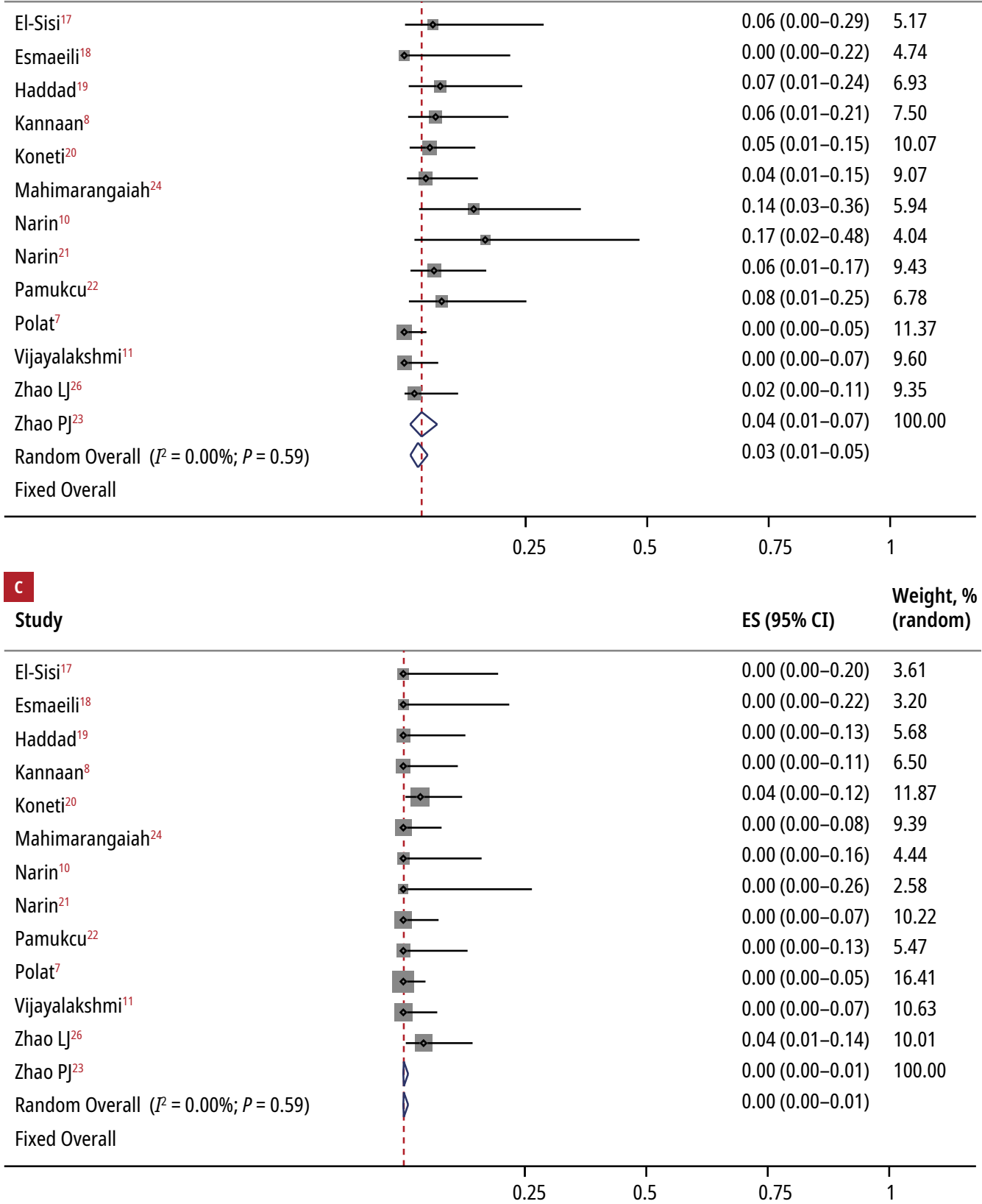

FIGURE 2 Forest plots of residual shunts (B) and postoperative aortic regurgitations (C) Abbreviations: ES, effect size

regurgitations [TRs]). 7,19,20,21,26 The pooled estimate of the AR rate was $0 \%$ (95\% CI, $0 \%-1 \% ; I^{2}=0 \%$ ), and that of the TR rate was $1 \%$ (95\% CI, $0 \%-3 \%$; $I^{2}=48.34 \%$ ). (FIGURE 2 C)

Other complications Device embolism was found in 3 patients only: 2 patients had the device removed percutaneously and 1 surgically. None of the patients exhibited reduced cardiac systolic function or device thrombosis. A total of $7 \mathrm{pa}-$ tients were converted to conventional surgical repair due to device failure (1 patient), significant RS (1 patient), device embolism (1 patient), and severe AR ( 2 patients). In 2 cases, the device was removed percutaneous because of device embolization.

Heterogeneity analysis Even though all the $I^{2}$ of the results were below $50 \%$, in our opinion, the type of VSD is an important factor. Hence, we performed a subgroup analysis by VSD type and divided all studies into the pmVSD group and the group with various types of VSD to explore the heterogeneity of the RS outcome $\left(I^{2}=47.26 \%\right) . I^{2}$ was $20.13 \%$ for the former and $63.37 \%$ for the latter. These results suggest that VSD type might be a source of heterogeneity. Sensitivity analyses were explored by excluding 
each study separately to validate the stability of the RS rate; all the results showed that there was no significant difference. In addition, the fixedeffects model was also performed with no significant difference found comparing to the random-effects model.

Publication bias Statistical evidence of publication bias was detected by Egger and Begg tests as well as an Egger graph (FIGURE 3). There was no significant publication bias for success rate, $\mathrm{cAVB}$, postoperative $\mathrm{AR}$ and TR, as evidenced by the respective Egger test $P$ values of $0.53,0.722,0.393$, and 0.377 . For RS, the $P$ value was 0.044 by the Begg test 0.017 by the Egger test. Further, we used the trim-and-fill method, after filling 3 studies, the $95 \%$ CI of the pooled RS rate results was unstable, which suggested publication bias (FIGURE 3D).

A

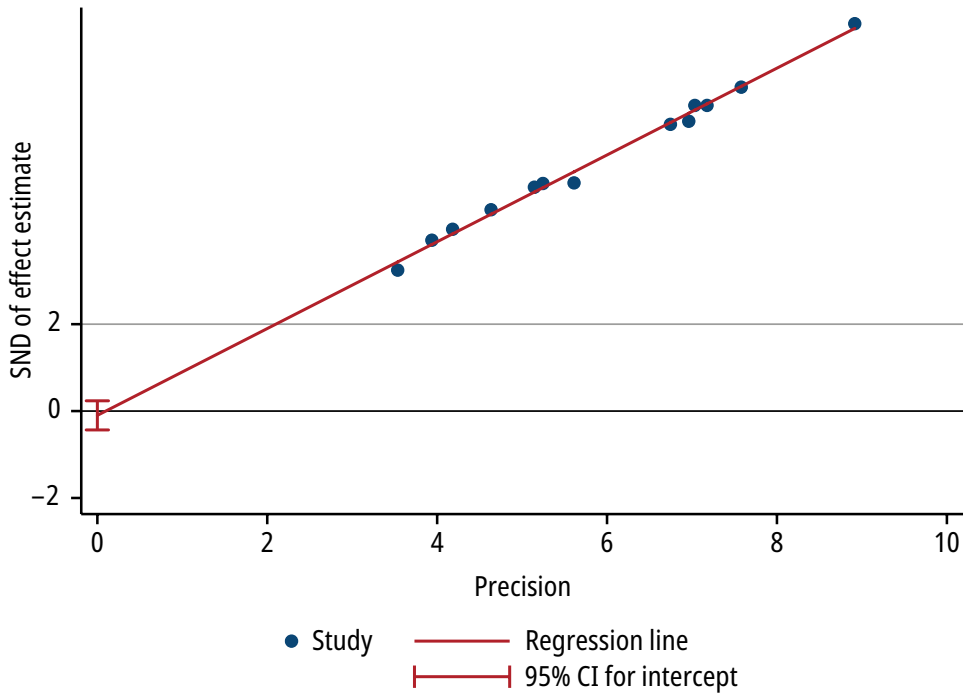

B

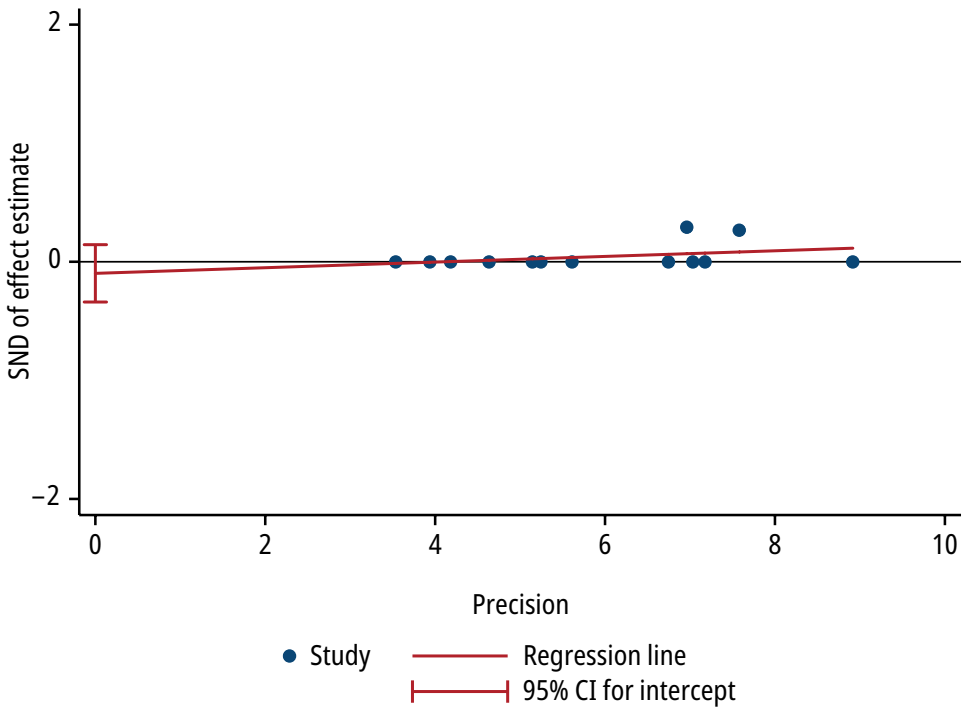

FIGURE 3 Egger graphs of successful outcome (A) and postoperative aortic regurgitations (B) Abbreviations: SND, standard normal deviation
DISCUSSION Perimembranous ventricular septal defect is the most common anomaly in congenital heart disease, accounting for almost one-fifth of all defects. ${ }^{27,28}$ Percutaneous closure has become an alternative for surgical closure in the majority of cases due to the following advantages: reduced operation time, short hospital stay, low cost, ability to avoid sternotomy and cardiopulmonary bypass, decreased blood loss and pain, and faster recovery and return to normal activities. ${ }^{3,29}$ However, percutaneous closure is considered to be safer in mVSD than in pmVSD. In fact, the proximity of the margins of pmVSD to the conduction system and aortic valves increases the risk of adverse events related to percutaneous closure, leading to several complications. ${ }^{30}$ Our study included $37 \mathrm{mVSD}$, 437 pmVSD, and a single hybrid VSD. The results showed that using the ADO II for VSD closure has a very high success rate and a relatively low incidence of complications.

Retrograde delivery of the Amplatzer Duct Occluder II improves surgical success When the VSD is small, the ventricular septum shunts from left to right; part of the pmVSD with an aneurysm has a small and diffuse shunt on the right ventricular surface, whereas part of the slender tubular mVSD is tortuous in the interventricular septum. Traditional VSD interventional therapy involves establishing an arteriovenous track, inserting the catheter from the femoral vein end to the ascending aorta, placing the catheter into the left ventricle, and finally using an anterograde release method to release the occluder. The surgical steps are tedious, especially for small or tortuous VSD, and it is difficult to pass the conventional occluder through the delivery sheath. ${ }^{26,31}$

A meta-analysis by Santhanam et $\mathrm{al}^{32}$ examined the outcomes of the use of any type of device (other than the early umbrella / Rashkind) to block pmVSD, with a success rate of $97.8 \%$ (95\% CI, 96.8\%-98.6\%). Notably, this analysis involved 54 studies with a total of 6762 patients, but only approximately $2 \%$ of patients ( 6 study with 121 patients) in whom the ADO II was used were included. Coincidentally, a meta-analysis by Hong et $\mathrm{al}^{29}$ including 15 studies with 1368 patients examined the outcomes of the use of any type of device, with a pooled success rate of 0.95 $\left(I^{2}=86.2 \% ; P<0.001\right)$. Our study showed that the success rate of the ADO II was much higher than that reported by Santhanam et al, ${ }^{32}$ a study that incorporated all types of devices. Overall, patients may benefit from the unique design of the ADO II, which is conducive to an easier and faster operation.

The ADO II is a double-disc device connected by the waist; it is made of nitinol alloy wire without a high-molecular-weight polymer filler. The supporting TorqVue LP delivery sheath 


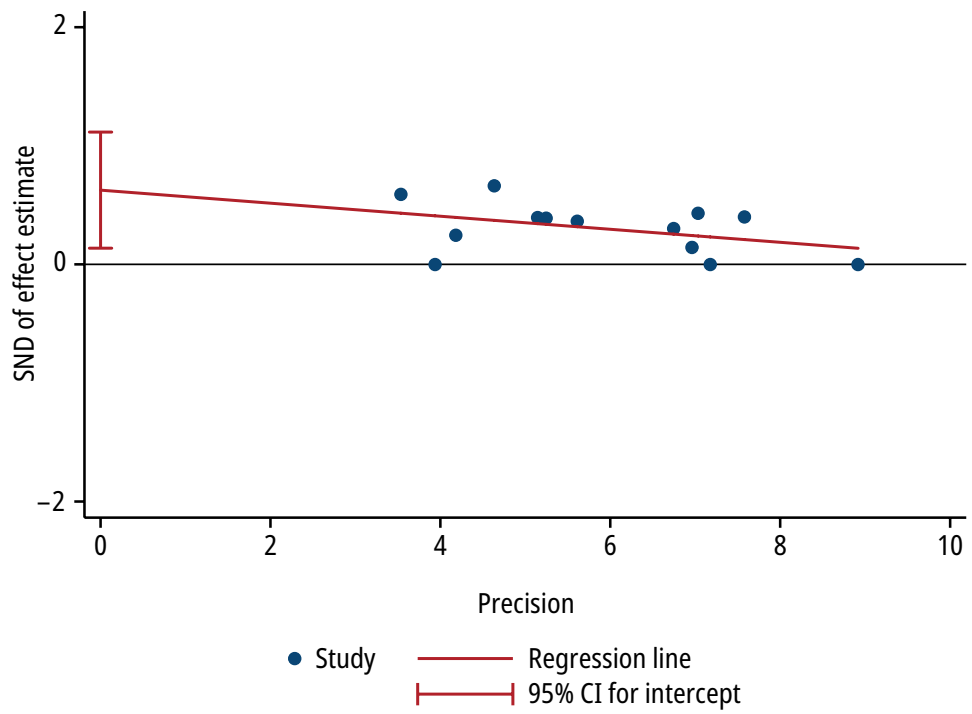

D

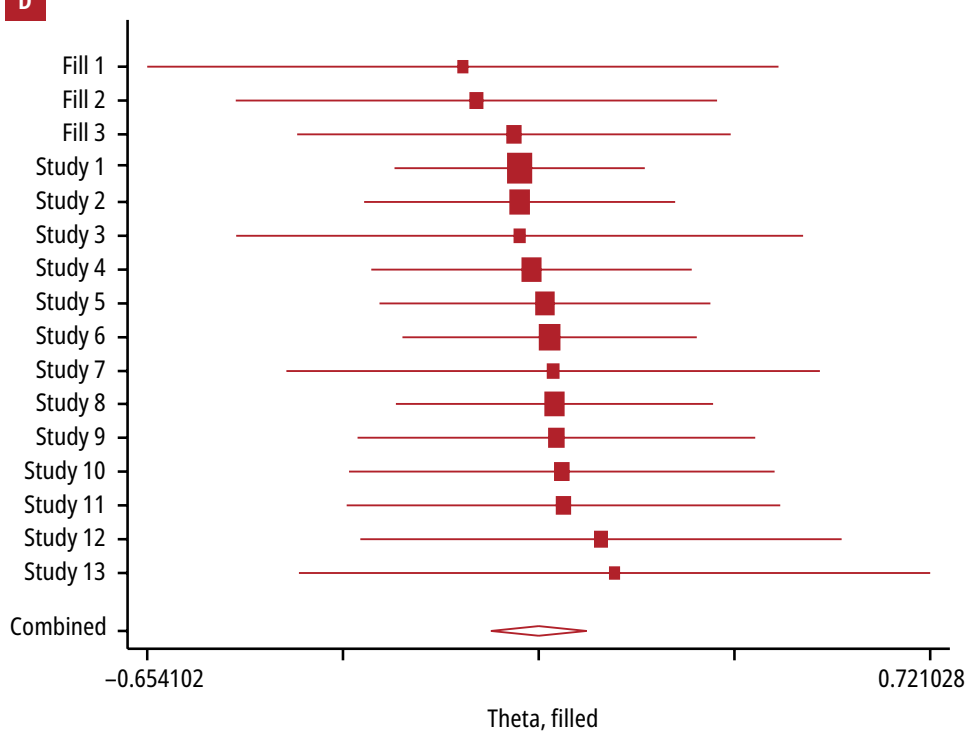

FIGURE 3 C - Egger graph of residual shunts; D - graph using the trim-and-fill method based on residual shunts

Abbreviations: SND, standard normal deviation

tube has a small diameter (4F-5F); hence, there is less damage to the vessel wall, enabling retrograde placement through the artery. Moreover, establishment of an arteriovenous track is not needed, and there are fewer consumables (saving a set of gooseneck trap consumables). Furthermore, the surgery is simpler, with a shorter time as well as less mechanical damage and intraoperative blood loss. ${ }^{6,19}$

Soft and elastic design of Amplatzer Duct Occluder II reduces surgical complications According to Santhanam et al, ${ }^{32}$ the most common complication during VSD occlusion is RS (pooled rate, $15.9 \%$; 95\% CI, 10.9\%-21.5\%). Other complications included arrhythmia (pooled rate, $10.3 \%$; $95 \%$ CI, $8.3 \%-12.4 \%$ ) and valve dysfunction (pooled rate, $4.1 \%$; $95 \% \mathrm{CI}, 2.4 \%-6.1 \%$ ), and the incidence of cAVB was $1.1 \%$ (95\% CI, 0.5\%-1.9\%).
In the meta-analysis of pmVSD by Hong et al, ${ }^{29}$ the pooled rate of postoperative RS was $2 \%(95 \%$ CI, $\left.1 \%-3 \% ; I^{2}=87.3 \% ; P<0.001\right)$. The pooled estimated rate of severe complications was $7.4 \%$ (95\% CI, $\left.4.6 \%-10.2 \%, I^{2}=30.5 \% ; P=0.13\right)$; the pooled incidence of $\mathrm{cAVB}$ was $0.2 \%$ (95\% CI, $0 \%-0.5 \%$; $\left.I^{2}=0 \% ; P=0.58\right)$. We reported a lower incidence of complications than Santhanam et $\mathrm{al}^{32}$ and Hong et al. ${ }^{29}$

Residual shunts Although an oversized device will lead to more damage to adjacent tissues, an undersized device may increase the embolic rate of the device and RSs. ${ }^{18}$ In the follow-up of other conventional blockers, the occurrence of RSs was found to be much lower than that observed immediately after the device was closed, indicating that time is critical for small RS closure. ${ }^{29,30,32,33}$ The population of the ADO II study in this meta-analysis included all patients with defects less than $6.5 \mathrm{~mm}$, small VSD shunt volumes, and smaller RSs after surgery, and some of them closed spontaneously during follow-up.

The waist of the ADO II is easily stretchable, with the waist diameter ranging from 3 to $6 \mathrm{~mm}$. Unlike the cylindrical waist of other occluders, the ADO II's waist has a spherical curvature. Therefore, in VSD with a membranous aneurysm, it enables a closer connection to the aneurysm and defect. Hence, the incidence of RS associated with the ADO II is low.

In addition, the ADO II with its unique waist structure can also release the occluder for the pmVSD without the aneurysm, which exerts a greater tension on the defect than other occluders, closely blocking the VSD, and has a low probability of participating in the shunt.

Moreover, the ADO II has a narrower waist and is made of softer material than other occluders, preventing it from affecting valves, especially the aortic valve. Due to the low probability of new valve regurgitation after the surgery, the stable hemodynamic state in the cardiac cavity is more conducive to endothelialization and endocardial formation, so even if a small amount of RS occurs, it can spontaneously disappear after a few weeks or months. 29,30,32,33

Complete atrioventricular block Complete atrioventricular block is a major challenge for transcatheter closure of pmVSD. ${ }^{11}$ It can occur during surgery or months and years afterward. ${ }^{30}$ It may be reversible or persistent, and persistent $\mathrm{CAVB}$ requires permanent pacing. Although the mechanism of cAVB is unknown, possible causes are as follows. First, compression of the device on both sides of the disc leads to injury of the conduction system. Then, mechanical stimulation is performed during the operation. Additionally, most pmVSDs lack the myoseptum surrounding the membrane-forming area, and the membrane-septal residue itself often 
compensatively develops into a pseudoseptal aneurysm in an attempt to close the defect. ${ }^{33-35}$

Consequently, in most cases, there is no conducting tissue at the top of the VSD, and it is less vulnerable to damage from device extrusion. However, in pmVSD without pseudoseptal aneurysm, the marginal portion of the defect consists of fibrous tissue from the continuous site of the tricuspid valve and the aortic valve, and the Hiss bundle passes through the posterior lower margin of pmVSD. Therefore, the heart conduction system is easily affected when the device is extruded, especially when the size of the blocker is overly large. Haddad et $\mathrm{al}^{19}$ and Vijayalakshmi et al ${ }^{11}$ claimed that the unique design of the ADO II makes it softer than other conventional devices and minimizes radial stress on the conduction system. It has also been reported that the pressure on ventricular septum tissue is small, tissue edema is light, and that heart block after surgery rarely occurs..$^{35,36}$

Valvular regurgitation The local anatomical structure of pmVSD is complex and adjacent to the tricuspid and aortic valves. Hence, inappropriate device selection during interventional treatment can easily result in valve insufficiency. The disc surface of the ADO II is symmetrical and $3 \mathrm{~mm}$ larger than that of the waist. Therefore, the distance between the defect upper margin and the aortic valve should be greater than $3 \mathrm{~mm}$ to avoid aortic valve regurgitation. ${ }^{8,10}$ Nevertheless, one of the studies (51 cases of the ADO II) included in our meta-analysis concluded that a subaortic residual length of $2 \mathrm{~mm}$ or greater was safe for the application of the ADO II. ${ }^{26}$

Due to the long waist of the ADO II, the surgeon needs to avoid excessive pulling of the occluder into the right ventricle, which could result in tricuspid dysfunction. Vijayalakshmi et $\mathrm{al}^{11}$ claimed that the ADO II is tailored to close the Gerbode defect because the central cylinder fits the defect and the soft disc without polyester material does not impinge on the mitral or tricuspid valve.

In conclusion, based on the summary of existing studies, we believe that the recommended application range of the ADO II for VSD is as follows: diameter from 3 to $6 \mathrm{~mm}$, pmVSD and mVSD (with or without aneurysm), and distance from the edge of the defect to the aortic valve of $2 \mathrm{~mm}$ or greater. $7,8,10,11,17-24$

Innovation and limitations To the best of our knowledge, our study is the first systematic review and meta-analysis to collate the outcomes of VSD closure with the ADO II. Nevertheless, this study had some limitations of note.

First, for ethical reasons and informed consent requirements, the included studies could not be treated blindly and had no blank control group. In addition, most of the included studies were retrospective and with small samples, and thus, it was difficult for the NOS score to exceed 6 . We believe that a low to medium NOS score is an unavoidable problem in most cohort studies of congenital heart disease. Second, the mean follow-up of the included studies ranged from 6 to 40 months, with most being short- and medium-term follow-ups. Third, different studies defined valve regurgitation differently. Indeed, a number of studies recorded only moderate or new valve regurgitation postoperatively, but others registered all types (preoperative and postoperative). As a consequence, we only included cases of newly occurring or aggravated valve regurgitation after surgery in the statistics, which may have caused underestimation of the result. Fourth, as most of the studies involving mVSD and pmVSD did not group them separately, we were unable to compare outcomes between pmVSD and mVSD. Finally, publication bias in this meta-analysis should be taken into consideration.

Conclusions This meta-analysis indicates the successful use of the ADO II, with a low incidence of RSs, AR, and TR. The ADO II may be a cost-effective device option in children with relatively small defects. These good outcomes may be limited by the number of enrolled patients, and more detailed observations in larger samples and long-term follow-up data are needed for further analysis.

\section{ARTICLE INFORMATION}

ACKNOWLEDGMENTS This study was supported by the Guangdong Science and Technology Plan Project (no. 2017zC0180; to PS).

CONFLICT OF INTEREST None declared.

OPEN ACCESS This is an Open Access article distributed under the terms of the Creative Commons Attribution-NonCommercial-NoDerivatives $4.0 \mathrm{In}$ ternational License (CC BY-NC-ND 4.0), allowing third parties to download articles and share them with others, provided the original work is properly cited, not changed in any way, distributed under the same license, and used for noncommercial purposes only. For commercial use, please contact the journal office at kardiologiapolska@ptkardio.pl.

HOW TO CITE Cen H, Peng B, Li J, et al. Efficacy and safety of the Amplatzer Duct Occluder II for ventricular septal defect closure: a meta-analysis. Kardiol Pol. 2021; 79: 401-409. doi:10.33963/KP.15762

\section{REFERENCES}

1 Lopez L, Houyel L, Colan SD, et al. Classification of ventricular septal defects for the eleventh iteration of the international classification of diseases-striving for consensus: a report from the International Society for Nomenclature of Paediatric and Congenital Heart Disease. Ann Thorac Surg. 2018; 106: 1578-1589.

2 Kidd L, Driscoll DJ, Gersony WM, et al. Second natural history study of congenital heart defects. Results of treatment of patients with ventricular septal defects. Circulation. 1993; 87: I38-I51.

3 Saurav A, Kaushik M, Mahesh Alla V, et al. Comparison of percutaneous device closure versus surgical closure of peri - membranous ventricular septal defects: a systematic review and meta-analysis. Catheter Cardiovasc Interv. 2015; 86: 1048-1056.

4 Ghaderian M, Merajie M, Mortezaeian H, et al. Efficacy and safety of using Amplatzer Ductal Occluder for transcatheter closure of perimembranous ventricular septal defect in pediatrics. Iran J Pediatr. 2015; 25: e386.

5 Epcacan S, Bulut M0, Kaya Y, et al. Characteristics and transcatheter closure of patent ductus arteriosus in patients living at moderate to high altitude in Eastern Anatolia. Turk Kardiyol Dern Ars. 2019; 47: 431-439. 
6 Kouakou NYN, Song J, Huh J, Kang IS. The experience of transcatheter closure of postoperative ventricular septal defect after total correction. J Cardiothorac Surg. 2019; 14: 104.

7 Polat TB, Turkmen E. Transcatheter closure of ventricular septal defects using the Amplatzer Duct Occluder II device: a single-center experience. Postepy Kardiol Interwencyjnej. 2016; 12: 340-347.

8 Kanaan M, Ewert P, Berger F, et al. Follow-up of patients with interventional closure of ventricular septal defects with Amplatzer Duct Occluder II. Pediatr Cardiol. 2015; 36: 379-385.

9 Ergene 0, Kahya Eren N, Nazli C, et al. Percutaneous closure of perimembranous ventricular septal defects associated with septal aneurysm in adults. Turk Kardiyol Dern Ars. 2015; 43: 699-704.

10 Narin N, Baykan A, Pamukcu 0, et al. ADO II in percutaneous VSD closure in pediatric patients. J Interv Cardiol. 2015; 28: 479-484.

11 Vijayalakshmi IB, Narasimhan C, Singh B, Manjunath CN. Treatment of congenital non-ductal shunt lesions with the Amplatzer Duct Occluder II. Catheter Cardiovasc Interv. 2013; 89: E185-E193.

12 Moher D, Liberati A, Tetzlaff J, et al. Preferred reporting items for systematic reviews and meta-analyses: the PRISMA statement. Ann Intern Med. 2009; 151:264-269.

13 Grygier M, Sabiniewicz R, Smolka G, et al. Percutaneous closure of atrial septal defect: a consensus document of the joint group of experts from the Association of Cardiovascular Interventions and the Grown-Up Congenital Heart Disease Section of the Polish Cardiac Society. Kardiol Pol. 2020; 78: 1066-1083.

14 Luchini C, Stubbs B, Solmi M, Veronese N. Assessing the quality of studies in meta-analyses: advantages and limitations of the Newcastle Ottawa Scale. World J Meta-Anal. 2017; 5: 80-84.

15 Harbord RM, Harris RJ, Sterne JAC. Updated tests for small-study effects in meta-analyses. Stata J. 2009; 9: 197-210.

16 Duval S, Tweedie R. Trim and fill: A simple funnel-plot-based method of testing and adjusting for publication bias in meta-analysis. Biometrics. 2000; 56 : 455-63.

17 El-Sisi A, Sobhy R, Jaccoub V, Hamza H. Perimembranous ventricular septal defect device closure: choosing between Amplatzer Duct Occluder I and II. Pediatr Cardiol. 2017; 38: 596-602

18 Esmaeili A, Behnke-Hall K, Schrewe R, Schranz D. Percutaneous closure of perimembranous ventricular septal defects utilizing almost ideal Amplatzer Duct Occluder II: why limitation in sizes? Congenit Heart Dis. 2018; 14: 389-395.

19 Haddad RN, Daou L, Saliba Z. Device closure of perimembranous ventricular septal defect: choosing between Amplatzer Occluders. Front Pediatr. 2019; 7: 300.

20 Koneti NR, Sreeram N, Penumatsa RR, et al. Transcatheter retrograde closure of perimembranous ventricular septal defects in children with the Amplatzer Duct Occluder II device. J Am Coll Cardiol. 2012; 60: 2421-2422.

21 Narin N, Pamukcu 0, Tuncay A, et al. Percutaneous ventricular septal defect closure in patients under 1 year of age. Pediatr Cardiol. 2018; 39: 1009-1015.

22 Pamukcu 0, Narin N, Baykan A, et al. Mid-term results of percutaneous ventricular septal defect closure with Amplatzer Duct Occluder-II in children. Cardiol Young. 2017; 27: 1726-1731.

23 Zhao PJ, Yu ZQ, Gao W et al. Efficacy of the transcatheter closure of perimembranous and muscular ventricular septal defects with the Amplatzer duct occluder II. Zhonghua Xin Xue Guan Bing Za Zhi. 2012; 40: 817-820.

24 Mahimarangaiah J, Subramanian A, Hemannasetty SK, et al. Transcatheter closure of perimembranous ventricular septal defects with ductal occluders. Cardiol Young. 2015; 25: 918-926.

25 Koneti NR, Verma S, Bakhru S, et al. Transcatheter trans-septal antegrade closure of muscular ventricular septal defects in young children. Catheter Cardiovasc Interv. 2013; 82: E500-E506.

26 Zhao LJ, Han B, Zhang JJ, et al. Transcatheter closure of congenital perimembranous ventricular septal defect using the Amplatzer duct occluder 2. Cardiol Young. 2017; 28: 447-453.

27 Jian Y, Lifang Y, Shiqiang Y, et al. Transcatheter versus surgical closure of perimembranous ventricular septal defects in children: a randomized controlled trial. J Am Coll Cardiol. 2014; 63: 1159-1168

28 Schmaltz AA, Neudorf U, Sack S, Galal 0. Interventions in congenital heart diseases and their sequelae in adults. Herz. 1999; 24: 293-306.

29 Hong ZN, Chen Q, Huang LQ, Cao H. A meta-analysis of perventricular device closure of perimembranous ventricular septal defect. J Cardiothorac Surg. 2019; 14: 119 .

30 Shah JH, Saraiya SP, Nikam TS, Jha MJ. Transcatheter device closure of perimembranous ventricular septal defect in pediatric patients: long-term outcomes. Heart Views. 2020; 21: 17-21.

31 Devendran V, Koneti NR, Jesudian V. Transcatheter closure of multiple perimembranous ventricular septal defects with septal aneurysm using two overlapping Amplatzer Duct Occluders II. Pediatr Cardiol. 2013; 34: 1963-1965.

32 Santhanam H, Yang L, Chen Z, et al. A meta-analysis of transcatheter device closure of perimembranous ventricular septal defect. Int J Cardiol. 2018; 254: 75-83.
33 Li P, Zhao XX, Zheng X, Qin YW. Arrhythmias after transcatheter closure of perimembranous ventricular septal defects with a modified double-disk occluder: early and long-term results. Heart Vessels. 2012; 27: 405-410.

34 Yip WC, Zimmerman F, Hijazi ZM. Heart block and empirical therapy after transcatheter closure of perimembranous ventricular septal defect. Catheter Cardiovasc Interv. 2005; 66: 436-441.

35 Carminati M, Butera G, Chessa M, et al. Transcatheter closure of congenital ventricular septal defects: results of the European Registry. Eur Heart J. 2007; 28: 2361-2368.

36 El Said HG, Bratincsak A, Gordon BM, Moore JW. Closure of perimembranous ventricular septal defects with aneurysmal tissue using the Amplazter Duct Occluder I: lessons learned and medium term follow up. Catheter Cardiovasc Interv. 2012; 80: 895-903. 